\title{
Homogenization of ferromagnetic energies on Poisson random sets in the plane
}

\author{
ANDREA BRAIDES \\ Dipartimento di Matematica, Università di Roma 'Tor Vergata' \\ via della Ricerca Scientifica, 00133 Rome, Italy \\ AndRey Piatnitski \\ The Arctic University of Norway, UiT, Campus Narvik, \\ P.O. Box 385, Narvik 8505, Norway \\ and \\ Institute for Information Transmission Problems of RAS, \\ 127051 Moscow, Russia
}

\begin{abstract}
We prove that by scaling nearest-neighbour ferromagnetic energies defined on Poisson random sets in the plane we obtain an isotropic perimeter energy with a surface tension characterised by an asymptotic formula. The result relies on proving that cells with 'very long' or 'very short' edges of the corresponding Voronoi tessellation can be neglected. In this way we may apply Geometry Measure Theory tools to define a compact convergence, and a characterisation of metric properties of clusters of Voronoi cells using limit theorems for subadditive processes.
\end{abstract}

Keywords. Poisson random sets, ferromagnetic energies, homogenization, firstpassage percolation, sets of finite perimeter, interfacial energies, $\Gamma$-convergence.

\section{Introduction}

In this paper we study a prototypical model of pair-interaction energies on Poisson random sets in the plane. These energies are a random extension of nearest-neighbour 'ferromagnetic' systems defined on Bravais lattices, whose overall behaviour is that of an interfacial energy [20, 1]. The possibility of lattice approximations for arbitrary interfacial energies makes the analysis of ferromagnetic energies relevant for numerical approximations and modeling issues (we refer to [15] for optimal constructions on regular lattices, available even with constraints on the interaction potentials). Surface energies are an important building block in the study of general functionals defined on more complex spaces of functions of bounded variation, passing through the generalization to 
functions with a discrete number of values [5] and using the latter to approximate arbitrary functions by coarea-type arguments (see e.g. 4]). Furthermore, the study of energies involving bulk and surface part can often be decoupled in the analysis of each part, which justifies the analysis of surface energies separately also in that context (see [14, 21] and the recent advances in the analysis and derivation of complex energies from discrete systems in [6, 7]). The present contribution can be then viewed as a step towards the extension of the analysis of discrete systems producing bulk and surface integrals to general random distribution of points. The simplest case of parameters taking only two values (equivalently, characteristic functions) will allow us to concentrate on the basic geometric features of the underlying discrete environment.

Discrete energies with randomness producing surface effects have been previously considered under various hypotheses. Results on regular lattices with random interactions comprise: random weak membrane models in [17, random ferromagnetic energies with positive coefficients in 19 and ferromagnetic energies with a random distribution of degenerate coefficients in 18. Stochastic lattices have been considered under the hypothesis that sites be distributed in such a way that no 'great holes' of 'concentration of sites' may occur, so that we obtain uniform upper and lower estimates for the size of the Voronoi cells of the underlying tessellation, which implies that these lattices can be treated in average as a regular periodic lattice (see $8,2,2,13$, 13). Our focus is precisely in avoiding such an hypothesis considering points distributed according to a Poisson point process in the plane (Poisson random set). We denote by $\mathcal{N}$ such a set of points and by $\mathcal{E}$ the set of the edges of the underlying Delaunay triangulation, which are identified with pairs of points $(i, j)$ in $\mathcal{N} \times \mathcal{N}$ (the nearest neighbours in $\mathcal{N}$ ). The energy we consider can be viewed as defined on subsets $\mathcal{I}$ of $\mathcal{N}$ by

$$
E(\mathcal{I})=\#\{(i, j) \in \mathcal{E}: i \in \mathcal{I}, j \notin \mathcal{I}\} .
$$

Note that the same energy can be interpreted as the number of edges of the boundary of the set

$$
A_{\mathcal{I}}=\bigcup_{i \in \mathcal{I}} C_{i},
$$

where $C_{i}$ is the cell of the Voronoi tessellation containing the point $i \in \mathcal{N}$. Another way to write the same energy is by identifying each set $\mathcal{I}$ with a (scalar) spin function parameterized by indices in $\mathcal{N}$ and defined by $u_{i}^{\mathcal{I}}=1$ if $i \in \mathcal{I}$ and $u_{i}^{\mathcal{I}}=-1$ if $i \notin \mathcal{I}$, so that we may rewrite $E(\mathcal{I})$ as depending on $u^{\mathcal{I}}$, setting

$$
E\left(u^{\mathcal{I}}\right)=\frac{1}{8} \sum_{i, j \in \mathcal{N}}\left(u_{i}-u_{j}\right)^{2}=\frac{1}{4} \sum_{i, j \in \mathcal{N}}\left|u_{i}-u_{j}\right|,
$$

the factors coming from double counting and the fact that $\left|u_{i}-u_{j}\right| \in\{0,2\}$. Conversely, we may take this as the definition of the energy, and correspondingly pass to subsets of $\mathcal{N}$ by noting that $E\left(\mathcal{I}_{u}\right)=E(u)$, where $\mathcal{I}_{u}=\left\{I \in \mathcal{N}: u_{i}=1\right\}$.

In order to describe the overall properties of $E$ we perform a discrete-tocontinuum analysis through a scaling procedure. We intoduce a small parameter 
$\varepsilon>0$ and consider the scaled energy $E_{\varepsilon}$ defined on subsets of $\varepsilon \mathcal{E}$ by

$$
E_{\varepsilon}(\mathcal{I})=\varepsilon \#\{(i, j) \in \varepsilon \mathcal{E}: i \in \mathcal{I}, j \notin \mathcal{I}\},
$$

which again can be interpreted as $\varepsilon$ times the number of edges of the boundary of the scaled set

$$
A_{\mathcal{I}}^{\varepsilon}=\varepsilon \bigcup_{i \in \mathcal{I}} C_{i / \varepsilon}
$$

Note that if we had a uniform upper and lower bound of the size of each of these edges, then $E_{\varepsilon}(\mathcal{I})$ would be comparable with the perimeter of $A_{\mathcal{I}}^{\varepsilon}$. In that case, given a family $\mathcal{I}_{\varepsilon}$ with equibounded $E_{\varepsilon}(\mathcal{I})$, the sets $A_{\varepsilon}=A_{\mathcal{I}_{\varepsilon}}^{\varepsilon}$ would be (locally) precompact in the sense of sets of finite perimeter; i.e., there would exist a set of finite perimeter $A$ such that, up to subsequences, $\left|\left(A_{\varepsilon} \triangle A\right) \cap Q\right| \rightarrow 0$ for any cube $Q$.

For Poisson random sets, the edges of Voronoi cells do not satisfy a uniform estimate. Nevertheless, very long or very short edges are in a sense negligible. Indeed, a result by Pimentel 34 implies that a path in which a large proportion of such sets is present must be 'short', and hence, by an isoperimetric argument encircle a 'small' set. Using this result, we can show that if $E_{\varepsilon}(\mathcal{I})$ is equibounded and $A_{\varepsilon}$ are defined above, then there exists families of sets $B_{\varepsilon}^{\prime}$ and $B_{\varepsilon}^{\prime \prime}$ such that $\left|B_{\varepsilon}^{\prime} \cup B_{\varepsilon}^{\prime \prime}\right| \rightarrow 0$ and the perimeter of the sets

$$
\left(A_{\varepsilon} \cup B_{\varepsilon}^{\prime}\right) \backslash B_{\varepsilon}^{\prime \prime}
$$

is equibounded. We deduce then that, up to subsequences, $A_{\varepsilon}$ still (locally) converge to a set of finite perimeter $A$.

We can then characterize the behaviour of the energies $E_{\varepsilon}$ by computing their $\Gamma$-limit with respect to this convergence. Note that, by the isotropy of Poisson random sets, if the limit is of perimeter type, it must be of the form

$$
F(A)=\tau_{0} \mathcal{H}^{1}(\partial A)
$$

i.e., a constant $\tau_{0}$ (the surface tension) times the perimeter of $A$ (in this notation $\partial A$ denotes the reduced boundary of $A$ ). The main issue is then to characterize such $\tau_{0}$ so as to adapt the discrete-to-continuum technique of [19, 18, to this case. A central observation is that the union of the boundaries of all Voronoi cells $C_{i}$ for which we have suitable outer and inner bounds determine a set which possesses a unique infinite connected component. We then introduce a parameter $\alpha>0$ that quantifies these bounds so that they become less and less stringent when $\alpha \rightarrow 0$. We denote by $\mathcal{V}_{\alpha}$ the union of boundaries of such Voronoi cells. The properties of $\mathcal{V}_{\alpha}$ are derived from percolation argument as in 18, 19, 17, and can be used to prove that a first-passage percolation formula holds for paths in $\mathcal{V}$ and at the same time permit to use the blow-up technique 24, 16] for proving a lower bound. An upper bound is finally shown by using the subadditive properties of the problems defining $\tau_{0}$.

It is worth noting that some of the results extend to arbitrary dimension (mainly, the compactness lemma for sets with equibounded energy), but the 
properties of regular Voronoi cells as stated and the characterization of $\tau_{0}$ with a first-passage percolation formula are particular of the planar case. The treatment of the asymptotic analysis of the energies in higher dimension will require different tools and homogenization formulas, which justify a separate treatment.

\section{Notation and statement of the results}

$\mathcal{L}^{2}(A)$ or $|A|$ denotes the 2-dimensional Lebesgue measure of a set $A, \mathbf{1}_{A}$ the characteristic function of the set $A, Q=[-1 / 2,1 / 2]^{2}$ the unit cube in $\mathbb{R}^{2}$.

\subsection{Poisson random sets}

$\mathcal{N}$ denotes a Poisson random set with intensity $\lambda>0$ in $\mathbb{R}^{2}$ defined on a probability space $(\Omega, \mathcal{F}, \mathbf{P})$. We refer for instance to 23 for the definition of a Poisson random set and its main properties. We recall that $\mathcal{N}$ is almost surely a locally finite subset of $\mathbb{R}^{2}$ such that

- for any bounded Borel set $B \subset \mathbb{R}^{2}$ the number of points in $B \cap \mathcal{N}$ has a Poisson law with parameter $\lambda|B|$

$$
\mathbf{P}\{\#(B \cap \mathcal{N})=n\}=e^{-\lambda|B|} \frac{(\lambda|B|)^{n}}{n !} ;
$$

- for any collection of bounded disjoint Borel subsets in $\mathbb{R}^{2}$ the random variables defined as the number of points of $\mathcal{N}$ in these subsets are independent.

We also assume that the probability space is equipped with a dynamical system $T_{x}: \Omega \mapsto \Omega, x \in \mathbb{R}^{2}$, and that for any bounded Borel set $B$ and any $x \in \mathbb{R}^{2}$ we have $\#((B+x) \cap \mathcal{N})(\omega)=\#(B \cap \mathcal{N})\left(T_{x} \omega\right)$. We recall that $T_{x}$ is a group of measurable measure preserving transformations in $\Omega$, also measurable as a function $T$. : $\Omega \times \mathbb{R}^{2} \mapsto \Omega$. We suppose that $T_{x}$ is ergodic. Further details can be found for instance in [28, Chapter 7].

In what follows, we only consider a Poisson random set with intensity 1 , since the results for as Poisson random set with intensity $\lambda$ may be obtained by considering the case with intensity 1 and then apply a scaling transformation $\mathcal{N} \longrightarrow \sqrt{\lambda} \mathcal{N}$

The cells of the Voronoi tessellation of $\mathcal{N}$ are denoted by

$$
C_{i}:=\left\{x \in \mathbb{R}^{2}:|x-i| \leq|x-j| \text { for all } j \in \mathcal{N}\right\}
$$

with $i \in \mathcal{N}$. Each $C_{i}$ thus defined is a polyhedral set; the set of edges of the Voronoi cells is denoted by $\mathcal{V}$. The set of the vertices of $C_{i}$ (or endpoints of elements of $\mathcal{V}$ ) is denoted by $\mathcal{N}^{*}$

Note that we may assume that each point in $\mathbb{R}^{2}$ belongs to at most three Voronoi cells or three elements of $\mathcal{E}$, since this is an event of probability 1.

The set of edges of the Delaunay triangulation of $\mathcal{N}$ is denoted by $\mathcal{E}$ and is identified with the set of pairs $(i, j)$ in $\mathcal{N} \times \mathcal{N}$ such that $C_{i}$ and $C_{j}$ share a common edge. 
We define a path of Voronoi cells as a collection $\left\{C_{i_{j}}: 1 \leq j \leq K\right\}$ such that $C_{i_{j}}$ and $C_{i_{j+1}}$ have an edge in common, or, equivalently, such that $\left(i_{j}, i_{j+1}\right) \in \mathcal{E}$ for all $j \in\{1, \ldots, K-1\}$. From the latter standpoint, we also talk of a path in $\mathcal{E}$. We say that such a path connects two sets $X$ and $Y$ if $X \cap C_{1} \neq \emptyset$ and $Y \cap C_{K} \neq \emptyset$. If $X=\{x\}$ and $Y=\{y\}$ then we simply say that the path connects $x$ and $y$.

\subsection{Asymptotic behaviour of ferromagnetic energies on Poisson random sets}

For future reference and comparison with the existing literature, we state our results in terms of energies on (scalar) spin functions, keeping in mind the possible alternative formulations as energies on sets or on set of points. The (scaled) ferromagnetic energy of the Poisson random set is defined on spin functions $u: \varepsilon \mathcal{N} \rightarrow\{-1,1\}$ by

$$
\begin{aligned}
E_{\varepsilon}(u) & =\frac{1}{8} \sum_{(i, j) \in \varepsilon \mathcal{E}} \varepsilon\left(u_{i}-u_{j}\right)^{2} \\
& =\frac{1}{2} \varepsilon \#\left\{(i, j) \in \varepsilon \mathcal{E}: u_{i} \neq u_{j}\right\} \\
& =\varepsilon \#\left\{(i, j) \in \varepsilon \mathcal{E}: u_{i}=1, u_{j}=-1\right\},
\end{aligned}
$$

where the scaling factor $\frac{1}{8}$ is due to double counting and to the fact that $\left(u_{j}-\right.$ $\left.u_{j}\right)^{2} \in\{0,4\}$. by

To each $u: \varepsilon \mathcal{N} \rightarrow\{-1,1\}$ we associate the (scaled) Voronoi set of $u$ defined

$$
V_{\varepsilon}(u)=\bigcup_{\left\{i: u_{i}=1\right\}} \varepsilon C_{i / \varepsilon},
$$

and the piecewise-constant interpolation (with underlying set $\varepsilon \mathcal{N}$ ), still denoted $u: \mathbb{R}^{2} \rightarrow\{-1,1\}$, defined by

$$
u(x)= \begin{cases}1 & \text { if } x \in V_{\varepsilon}(u) \\ -1 & \text { if } x \in \mathbb{R}^{2} \backslash V_{\varepsilon}(u) .\end{cases}
$$

Definition 2.1. We say that a family $u^{\varepsilon}: \varepsilon \mathcal{N} \rightarrow\{-1,1\}$ converges to a set $A$ if the piecewise-constant interpolations $u^{\varepsilon}$ converge to the function $\mathbf{1}_{A}-\mathbf{1}_{\mathbb{R}^{2}} \backslash A$ locally in $L^{1}\left(\mathbb{R}^{2}\right)$, or, equivalently, if $\mathbf{1}_{V_{\varepsilon}\left(u^{\varepsilon}\right)}$ converge to $\mathbf{1}_{A}$ locally in $L^{1}\left(\mathbb{R}^{2}\right)$.

The following compactness lemma justifies the use of the convergence in Definition 2.1 in the computation of the $\Gamma$-limit of $E_{\varepsilon}$ [11, 12. Note that the result cannot be directly deduced from the compactness property of sets of equibounded perimeter, since we cannot deduce the equiboundedness of the perimeters of the sets $V_{\varepsilon}\left(u^{\varepsilon}\right)$ from the equiboundedness of $E_{\varepsilon}\left(u_{\varepsilon}\right)$. 
Lemma 2.2 (compactness). Let $u^{\varepsilon}$ be such that $\sup _{\varepsilon} E_{\varepsilon}\left(u^{\varepsilon}\right)<+\infty$. Then, up to subsequences $u^{\varepsilon}$ converges to some set $A$ in the sense of Definition 2.1. Moreover, the limit set is a set of finite perimeter.

The compactness lemma above ensures that the domain of the $\Gamma$-limit of $E_{\varepsilon}$ be the family sets of finite perimeter in $\mathbb{R}^{2}$. The asymptotic behaviour of $E_{\varepsilon}$ will be described by an asymptotic formula similar to those encountered in first-passage percolation, involving minimal paths on $\mathcal{E}$ between points of $\mathbb{R}^{2}$. To that end we define for all $x \in \mathbb{R}^{2}$

$$
\pi_{0}(x)=\text { closest point of } \mathcal{N}^{*} \text { to } x .
$$

For almost all $x$ this point is uniquely defined. For the remaining points we choose one of the closest points of $\mathcal{N}^{*}$ to $x$. For $x, y \in \mathbb{R}^{2}$ we define

$$
m_{0}(x, y)=\min \left\{\#\left\{e_{k}\right\}:\left\{e_{k}\right\} \text { is a path in } \mathcal{E} \text { connecting } \pi_{0}(x) \text { and } \pi_{0}(y)\right\},
$$

where a path of segments (in our case edges in $\mathcal{V}$ ) connecting two points $\bar{x}$ and $\bar{y}$ is a collection of segments $\left[x_{k-1}, x_{k}\right]$ with $1 \leq k \leq K$ for some $K \in \mathbb{N}$ such that $x_{0}=\bar{x}$ and $x_{K}=\bar{y}$, and such that the related piecewise-linear curve is not self-intersecting.

Theorem 2.3 (homogenization theorem). Let $\mathcal{E}$ be a Poisson random set with intensity 1 . Then there exists a deterministic constant $\tau_{0} \in(0,+\infty)$ (the surface tension) such that almost surely the energies $E_{\varepsilon}$ defined in (8) $\Gamma$-converge to the energy $F(A)=\tau_{0} \mathcal{H}^{1}(\partial A)$, defined on sets of finite perimeter, with respect to the convergence in Definition 3.4. Furthermore the constant $\tau_{0}$ satisfies

$$
\tau_{0}=\lim _{t \rightarrow \infty} \frac{m_{0}((0,0),(t, 0))}{t}
$$

almost surely, where $m_{0}$ is given by (11).

The proof of this result will be the content of Section 4

Remark 2.4. By the scaling argument $\mathcal{N} \rightarrow \sqrt{\lambda} \mathcal{N}$, we deduce that if $\mathcal{E}$ is a Poisson random set with intensity $\lambda$ then the $\Gamma$-limit of the corresponding $E_{\varepsilon}$ is $\sqrt{\lambda} \tau_{0} \mathcal{H}^{1}(\partial A)$.

\section{Compactness}

This section is devoted to the proof of the Compactness Lemma 2.2 Even though we will use it in the planar case, we note that that result can be proved in any space dimension $d$ up to minor changes (see Remark 3.3 below).

$\Pi$ denotes the set of finite connected unions of Voronoi cells (here connected means that the corresponding set of edges of the Delaunay triangulation is connected). If $P \in \Pi$ we set

$$
\mathbf{A}(P)=\left\{z \in \mathbb{Z}^{2}:(z+Q) \cap P \neq \emptyset\right\} .
$$


Lemma 3.1 (Pimentel's polyomino lemma [34). Let $R>0$ and $\gamma>0$. Then there exists a deterministic constant $C$ such that for almost all $\omega$ there exists $\varepsilon_{0}=\varepsilon_{0}(\omega)>0$ such that if $P \in \Pi$ and $\varepsilon<\varepsilon_{0}$ satisfy

$$
P \cap \frac{R}{\varepsilon} Q \neq \emptyset, \quad \max \left\{\#\left\{i: C_{i} \subset P\right\}, \# \mathbf{A}(P)\right\} \geq \varepsilon^{-\gamma}
$$

then we have

$$
\frac{1}{C} \#\left\{i: C_{i} \subset P\right\} \leq \# \mathbf{A}(P) \leq C \#\left\{i: C_{i} \subset P\right\} .
$$

Note in particular that in the hypotheses of the lemma, we also have

$$
\min \left\{\#\left\{i: C_{i} \subset P\right\}, \# \mathbf{A}(P)\right\} \geq \frac{1}{C} \varepsilon^{-\gamma} .
$$

Further geometric properties of such Voronoi tessellations can be found in [22].

Lemma 2.2 will be a consequence of the following result.

Lemma 3.2 (compactness of Voronoi sets). Let $u^{\varepsilon}$ be such that $\sup _{\varepsilon} E_{\varepsilon}\left(u^{\varepsilon}\right)<$ $+\infty$. Then we can write

$$
V_{\varepsilon}\left(u^{\varepsilon}\right)=\left(A_{\varepsilon} \cup B_{\varepsilon}^{\prime}\right) \backslash B_{\varepsilon}^{\prime \prime},
$$

where $\left|B_{\varepsilon}^{\prime}\right|+\left|B_{\varepsilon}^{\prime \prime}\right| \rightarrow 0$, the family $\mathbf{1}_{A_{\varepsilon}}$ is precompact in $L_{\mathrm{loc}}^{1}\left(\mathbb{R}^{2}\right)$ and each its limit point is the characteristic function of a set of finite perimeter $A$, so that the same holds for $\mathbf{1}_{V_{\varepsilon}\left(u^{\varepsilon}\right)}$.

Proof. Since we reason locally, in order to ease the notation we assume that e.g. all $u^{\varepsilon}$ are identically -1 outside a fixed cube (or equivalently that $V_{\varepsilon}\left(u^{\varepsilon}\right)$ are contained in a fixed cube).

We fix $\gamma>0$ small enough. We subdivide $\partial V_{\varepsilon}\left(u^{\varepsilon}\right)$ into its connected components. We denote by $\mathcal{C}_{\varepsilon}^{\gamma,+}$ the family of such connected components $S$ with

$$
\#\left\{i \in \mathcal{N}: u_{\varepsilon i}=1, \varepsilon C_{i} \cap S \neq \emptyset\right\} \geq \varepsilon^{-\gamma} .
$$

Note that each such connected component can be identified with the set

$$
P=P(S)=\bigcup\left\{C_{i}: u_{\varepsilon i}=1, \varepsilon C_{i} \cap S \neq \emptyset\right\},
$$

which belongs to the set $\Pi$. We denote by $\mathcal{C}_{\varepsilon}^{\gamma,-}$ the family of the remaining connected components.

The first step will be to identify the small sets $B_{\varepsilon}^{\prime}$ and $B_{\varepsilon}^{\prime \prime}$ as the 'interior' of contours in $\mathcal{C}_{\varepsilon}^{\gamma,-}$ where the inner trace of $\mathbf{1}_{V_{\varepsilon}\left(u^{\varepsilon}\right)}$ is 0 and 1 , respectively. In this way the remaining set will have a boundary only composed of 'large' components from $\mathcal{C}_{\varepsilon}^{\gamma,+}$. This argument needs a little more formalization since we may have contours contained in other contours.

By the finiteness of the energy we have

$$
\# \mathcal{C}_{\varepsilon}^{\gamma,-} \leq \frac{C}{\varepsilon}
$$


Note that

$$
\#\left(\mathbf{A}\left(\frac{1}{\varepsilon} S\right)\right) \leq C \varepsilon^{-\gamma} \text { for every } S \in \mathcal{C}_{\varepsilon}^{\gamma,-}
$$

Indeed, otherwise $\#\left(\mathbf{A}\left(\frac{1}{\varepsilon} S\right)\right)>C \varepsilon^{-\gamma}>\varepsilon^{-\gamma}$, so that the hypotheses of Lemma 3.2 are satisfied and (13) implies that (15) holds, which gives a contradiction. Hence each $S \in \mathcal{C}_{\varepsilon}^{\gamma,-}$ is contained in a set with boundary at most of length $C \varepsilon^{1-\gamma}$. By an isoperimetric estimate, the measure of the bounded set sorrounded by each $S \in \mathcal{C}_{\varepsilon}^{\gamma,-}$ is $O\left(\varepsilon^{2-2 \gamma}\right)$. Hence, the total measure of such sets is $O\left(\varepsilon^{1-2 \gamma}\right)$.

Consider now each maximal $S \in \mathcal{C}_{\varepsilon}^{\gamma,-}$; i.e., which is not contained in any other bounded set whose boundary is another element in $\mathcal{C}_{\varepsilon}^{\gamma,-}$. For each such $S$, let $P$ be defined from $S$ by 16 . We have two cases, whether $\varepsilon P$ is interior to $S$ or not. We denote by $\mathcal{C}_{1, \varepsilon}^{\gamma,-}$ the first family, by $\mathcal{C}_{2, \varepsilon}^{\gamma,-}$ the second one, and define $B_{\varepsilon}^{\prime}$ as the union of the $\varepsilon C_{i / \varepsilon}$ in the interior of $S$ for some $S \in \mathcal{C}_{1, \varepsilon}^{\gamma,-}$ and such that $u_{i}^{\varepsilon}=1$, and $B_{\varepsilon}^{\prime \prime}$ as the union of the $\varepsilon C_{i / \varepsilon}$ in the interior of $S$ for some $S \in \mathcal{C}_{2, \varepsilon}^{\gamma,-}$ and such that $u_{i}^{\varepsilon}=-1$. If we set

$$
A_{\varepsilon}=\left(V_{\varepsilon}\left(u^{\varepsilon}\right) \backslash B_{\varepsilon}^{\prime}\right) \cup B_{\varepsilon}^{\prime \prime}
$$

then $\partial A_{\varepsilon}$ consists only of components in $\mathcal{C}_{\varepsilon}^{\gamma,+}$, and

$$
\left|B_{\varepsilon}^{\prime} \cup B_{\varepsilon}^{\prime \prime}\right| \leq C \varepsilon^{1-2 \gamma} .
$$

In order to prove the compactness of $A_{\varepsilon}$ we write $A_{\varepsilon}=A_{\varepsilon}^{\prime} \cup A_{\varepsilon}^{\prime \prime}$, where

$$
\begin{aligned}
& \left.A_{\varepsilon}^{\prime}=\bigcup\{(\varepsilon z+\varepsilon Q): \varepsilon z+\varepsilon Q) \subset \partial A_{\varepsilon}\right\} \\
& A_{\varepsilon}^{\prime \prime}=A_{\varepsilon} \backslash A_{\varepsilon}^{\prime}
\end{aligned}
$$

Note that

$$
\partial A_{\varepsilon}^{\prime} \subset \varepsilon \bigcup_{S \in \mathcal{C}_{\varepsilon}^{\gamma,+}} \partial \mathbf{A}(P(S))
$$

with $P(S)$ defined in (16). By Lemma 3.1 we have

$$
\mathcal{H}^{1}(\partial \mathbf{A}(P(S))) \leq C \#\left\{i \in \mathcal{N}: u_{\varepsilon i}=1, \varepsilon C_{i} \cap S \neq \emptyset\right\}
$$

Summing up over all $S \in \mathcal{C}_{\varepsilon}^{\gamma,+}$ we obtain

$$
\mathcal{H}^{1}\left(\partial A_{\varepsilon}^{\prime}\right) \leq C E_{\varepsilon}\left(u^{\varepsilon}\right) .
$$

Hence, the functions $\mathbf{1}_{A_{\varepsilon}^{\prime}}$ are locally precompact in $L^{1}\left(\mathbb{R}^{2}\right)$ by the precompactness of sets of equibounded perimeter [10, 33].

Again by Lemma 3.1 we have

$$
\left|A_{\varepsilon}^{\prime \prime}\right| \leq C \varepsilon^{2} \sum_{S \in \mathcal{C}_{\varepsilon}^{\gamma,+}} \# \mathbf{A}(P(S)) \leq C \varepsilon E_{\varepsilon}\left(u^{\varepsilon}\right) .
$$

This shows that $\left|A_{\varepsilon}^{\prime \prime}\right| \rightarrow 0$, and proves the claim. 
Remark 3.3. The previous compactness result holds in any dimension $d$ with minor changes in the proof, upon noting that Pimentel's lemma holds with

$$
\mathbf{A}(P)=\left\{z \in \mathbb{Z}^{d}:(z+Q) \cap P \neq \emptyset\right\}
$$

and $Q$ the coordinate unit cube in $\mathbb{R}^{d}$ [34].

Remark 3.4 (convergence in terms of the empirical measures). To each $u^{\varepsilon}$ : $\varepsilon \mathcal{N} \rightarrow\{-1,1\}$ we can associate the so-called empirical measure

$$
\mu\left(u^{\varepsilon}\right)=\sum_{\left\{i \in \varepsilon \mathcal{N}: u_{i}^{\varepsilon}=1\right\}} \varepsilon^{2} \delta_{i} .
$$

If $u^{\varepsilon}$ are such that $\sup _{\varepsilon} E_{\varepsilon}\left(u^{\varepsilon}\right)<+\infty$ and $u^{\varepsilon}$ converge to $A$ as in Definition 2.1. then the measures $\mu\left(u^{\varepsilon}\right)$ locally converge to the measure $\mathbf{1}_{A} \mathcal{L}^{2}$ with respect to the weak* convergence of measures. Thanks to Lemma 3.2. then these two convergences are equivalent.

To check the convergence of $\mu\left(u^{\varepsilon}\right)$, we first note that we may suppose that $\mu\left(u^{\varepsilon}\right) \rightarrow f \mathcal{L}^{2}$ for some $f: \mathbb{R}^{2} \rightarrow[0,1]$. It suffices to show that $f=0$ at almost every point of density 0 for $A$ (a symmetric argument then shows that $f=1$ at almost every point of density 1 for $A$ ).

For almost all such $x_{0}$ we have that

$$
\limsup _{\varepsilon \rightarrow 0}\left|V_{\varepsilon}\left(u^{\varepsilon}\right) \cap\left(x_{0}+\rho Q\right)\right|=o\left(\rho^{2}\right)
$$

and $\limsup _{\varepsilon \rightarrow 0} E_{\varepsilon}\left(u^{\varepsilon}, Q_{\rho}\right)=o(\rho)$, where we have set

$$
E_{\varepsilon}\left(u^{\varepsilon}, Q_{\rho}\right)=\frac{1}{2} \varepsilon \#\left\{(i, j) \in \varepsilon \mathcal{E}: u_{i}^{\varepsilon} \neq u_{j}^{\varepsilon}, i \text { or } j \in \rho Q\right\} .
$$

We may subdivide $V_{\varepsilon}\left(u^{\varepsilon}\right) \cap\left(x_{0}+\rho Q\right)$ into disjoint connected components:

$$
V_{\varepsilon}\left(u^{\varepsilon}\right) \cap\left(x_{0}+\rho Q\right)=\bigcup_{\#\left(P_{j} \cap \varepsilon \mathcal{N}\right) \leq \varepsilon^{-\gamma}} P_{j} \cup \bigcup_{\#\left(L_{k} \cap \varepsilon \mathcal{N}\right)>\varepsilon^{-\gamma}} L_{k},
$$

We may apply Lemma 3.1 to each $L_{k}$ to obtain

$$
\sum_{k} \varepsilon^{2} \#\left(L_{k} \cap \varepsilon \mathcal{N}\right) \leq C \varepsilon^{2} \sum_{k} \# \mathbf{A}\left(\frac{1}{\varepsilon} L_{k}\right) \leq C\left|V_{\varepsilon}\left(u^{\varepsilon}\right)\right|=o\left(\rho^{2}\right) .
$$

As for $P_{j}$ we have

$$
\#\left(\left\{P_{j}\right\}\right) \leq \frac{1}{\varepsilon} E_{\varepsilon}\left(u^{\varepsilon}, Q_{\rho}\right)=\frac{1}{\varepsilon} o(\rho), \quad \sum_{j} \#\left(P_{j} \cap \varepsilon \mathcal{N}\right) \leq \frac{1}{\varepsilon^{1+\gamma}} o(\rho) .
$$

In conclusion,

$$
\mu\left(u^{\varepsilon}\right)\left(x_{0}+\rho Q\right)=\varepsilon^{2} \#\left\{u_{i}^{\varepsilon}=1, i \in x_{0}+\rho Q\right\} \leq o\left(\rho^{2}\right)+\varepsilon^{1-\gamma} o(\rho) .
$$

Letting first $\varepsilon \rightarrow 0$ and then $\rho \rightarrow 0$ we prove the claim. 


\section{Proof of the Homogenization Theorem}

In this section we prove Theorem 2.3 first characterizing the surface tension and then computing the $\Gamma$-limit. Preliminarily, we introduce regular Voronoi cells and study their geometry.

\subsection{Geometry of clusters of regular Voronoi cells}

The surface tension characterizing the $\Gamma$-limit will be expressed by an asymptotic average length of minimal paths analogous to first-passage percolation formulas. A difficulty in our case is that in principle one of the end-points of such paths could be located in an 'exceptional region' where very small Voronoi cells accumulate. In order to treat this case, we first introduce regular Voronoi cells and study some percolation characteristics of the grid of such cells.

For $\alpha>0$ we set

$\mathcal{N}_{\alpha}^{0}=\left\{i \in \mathcal{N}: C_{i}\right.$ contains a ball of radius $\alpha$, diam $C_{i} \leq \frac{1}{\alpha}$, \# edges of $\left.C_{i} \leq \frac{1}{\alpha}\right\}$

the family of regular Voronoi cells with parameter $\alpha$. The following lemma describes some geometrical features of regular Voronoi tessellations.

Lemma 4.1 (a channel property of $\mathcal{N}_{\alpha}^{0}$ ). Let $\delta>0$. For every $T \in \mathbb{R}, \nu \in S^{1}$ and $x \in \mathbb{R}^{2}$ we define

$$
R_{T, \delta}^{\nu}(x)=\left\{x:\left|\left\langle x-x_{i}, \nu_{i}\right\rangle\right| \leq \delta T,\left|\left\langle x-x_{i}, \nu_{i}^{\perp}\right\rangle\right| \leq \frac{1}{2} T\right\} .
$$

Then there exist $\alpha_{0}, C_{\delta}>0$ such that a.s. there exists $T_{0}(\omega)>0$ such that for all $T>T_{0}(\omega)$ the rectangle $R_{T, \delta}^{\nu}(x)$ contains at least $C_{\delta} T$ disjoint paths of Voronoi cells $C_{i}$ with $i \in \mathcal{N}_{\alpha}^{0}$ connecting the two opposite sides of $R_{T, \delta}^{\nu}(x)$ parallel to $\nu$. This property is uniform as $x / T$ vary on a bounded set of $\mathbb{R}^{2}$.

Proof. Our arguments rely on the result known as channel property in the Bernoulli site percolation model in $\mathbb{Z}^{2}$. Denote $Q_{5 L}:=[-5 L, 5 L]^{2}$, and for $L, K, \alpha \in \mathbb{R}^{+}$and $j \in \mathbb{Z}^{2}$ denote by $\mathcal{E}(L, K, \alpha, j)$ the event that the following conditions are fulfilled:

$\left(\mathbf{c}_{1}\right)$ any square $[0, L]^{2}+L i$ with $i \in \mathbb{Z}^{2} \cap[-4.5,5.5]^{2}$ contains at least one point of $\mathcal{N}-10 j$

$\left(\mathbf{c}_{2}\right)$ the total number of points $\#\left((\mathcal{N}-10 L j) \cap Q_{5 L}\right)$ does not exceed $K$,

$\left(\mathbf{c}_{3}\right)$ the distance between any two points of $(\mathcal{N}-10 L j) \cap Q_{5 L}$ as well as the distance from any point of $(\mathcal{N}-10 L j) \cap Q_{5 L}$ to $\partial Q_{5 L}$ is greater than $2 \alpha$.

Letting $\xi_{j}$ be the characteristic function of $\mathcal{E}(L, K, \alpha, j)$ and considering the properties of the Poisson random set we conclude that $\xi_{j}, j \in \mathbb{Z}^{2}$, are i.i.d. 
random variables. For any $\gamma>0$ one can choose sufficiently large $L$ and $K$ and sufficiently small $\alpha>0$ so that

$$
\mathbf{P}(\mathcal{E}(L, K, \alpha, j))>1-\gamma .
$$

Indeed, the probability that any cube of size $L$ in $Q_{5 L}$ contains at least one point of the Poisson random set tends to 1 as $L \rightarrow \infty$. Then, given $L>0$, the probability that the number of points in $Q_{5 L}$ does not exceed $K$ tends to 1 as $K \rightarrow \infty$. The probability that in the cube $Q_{5 L}$ the smallest distance between two points is less than $\alpha$ goes to zero as $\alpha \rightarrow 0$. Finally, the probability that $\alpha$ neighbourhood of $\partial Q_{5 L}$ contains at least one point also goes to zero. Combining this relations we obtain the desired property.

For any two points $j^{\prime}, j^{\prime \prime} \in \mathbb{Z}^{2}$ such that $\left|j^{\prime}-j^{\prime \prime}\right|=1$ denote by $I_{j^{\prime}, j^{\prime \prime}}$ the segment $\left[10 L j^{\prime}, 10 L j^{\prime \prime}\right]$ in $\mathbb{R}^{2}$. If $\xi_{j^{\prime}}=\xi_{j^{\prime \prime}}=1$ then

(s1) any Voronoi cell $C_{i}$ that has a non-trivial intersection with $I_{j^{\prime}, j^{\prime \prime}}$ belongs to $\left(Q_{5 L}+10 L j^{\prime}\right) \cup\left(Q_{5 L}+10 L j^{\prime \prime}\right)$,

(s2) any such a cell $C_{i}$ contains a ball of radius $\alpha$,

(s3) the number of edges of each such $C_{i}$ is not greater than $K$.

In particular, due to $(\mathbf{s} 1)$ and $\left(\mathbf{c}_{2}\right)$, the total number of the cells $C_{i}$ having a non-empty intersection with $I_{j^{\prime}, j^{\prime \prime}}$ does not exceed $2 K$.

Statement (s1) can be justified as follows: Let $x^{\prime}$ be an arbitrary point of $I_{j^{\prime}, j^{\prime \prime}}$. Denote by $C_{i}$ the Voronoi cell that contains $x^{\prime}$ and by $x_{i}$ the corresponding point of the Poisson random set. Due to $\left(\mathbf{c}_{1}\right)$ we have $\left|x^{\prime}-x_{i}\right| \leq \sqrt{2} L$. Then any point $y \in \partial\left(\left(Q_{5 L}+10 L j^{\prime}\right) \cup\left(Q_{5 L}+10 L j^{\prime \prime}\right)\right)$ satisfies the inequality $\left|y-x_{i}\right| \geq(5-\sqrt{2}) L$. On the other hand, by $\left(\mathbf{c}_{1}\right)$ the distance of $y$ from $\mathcal{N}$ is not greater than $\sqrt{2} L$. This implies that $y \notin C_{i}$. Therefore, $C_{i} \subset\left(Q_{5 L}+10 L j^{\prime}\right) \cup\left(Q_{5 L}+10 L j^{\prime \prime}\right)$, and (s1) follows.

In a similar way one can show that for any $C_{i}$ that has a nontrivial intersection with $I_{j^{\prime}, j^{\prime \prime}}$ and any $x_{j} \in \mathcal{N}$ such that $C_{i}$ and $C_{j}$ have an edge in common we have $x_{j} \in\left(Q_{5 L}+10 L j^{\prime}\right) \cup\left(Q_{5 L}+10 L j^{\prime \prime}\right)$. In view of $\left(\mathbf{c}_{2}\right)$ this yields (s3).

Statement (s2) is an immediate consequence of $\left(\mathbf{c}_{3}\right)$.

Now the desired channel property follows from the well-known channel property in the Bernoulli site percolation model. For the reader convenience we formulate it here. Let $\eta_{j}, j \in \mathbb{Z}^{2}$, be a collection of i.i.d. random variables taking on the value 1 with probability $p$ and the value 0 with probability $(1-p)$. We say that $\left\{j_{i}\right\}_{i=1}^{M}$ is a 1 -path if $j_{i}$ and $j_{i+1}, i=1,2, \ldots, M-1$, are neighbouring points of $\mathbb{Z}^{2}$ and $\eta_{j_{i}}=1$ for all $i$. Then there exists $p_{\text {cr }} \in(0,1)$ such that for all $p>p_{\text {cr }}$ the following statement holds: for any $\delta>0$ there exists $\mathcal{K}_{\delta}>0$ such that for almost each $\omega \in \Omega$ there exists $T_{0}=T_{0}(\omega)>0$ such that any rectangle $R_{T, \delta}^{\nu}(x)$ with $T \geq T_{0}$ and $x \in[-T, T]^{2}$ contains at least $\mathcal{K}_{\delta}>0$ disjoint 1-paths connecting the two opposite sides of $R_{T, \delta}^{\nu}(x)$ parallel to $\nu$. We refer to [29] for further details.

It remains to choose $\gamma$ in (18) in such a way that $1-\gamma>p_{\text {cr. Labeling }}$ the squares $Q_{5 L}+10 j$ with the corresponding points $j \in \mathbb{Z}^{2}$ and recalling the 
just formulated channel property of the Bernoulli site percolation model with $\eta_{j}=\xi_{j}$ we obtain the desired statement.

From the proof of the previous lemma, in particular we obtain the following proposition.

Proposition 4.2. There exists $\alpha_{0}$ such that if $\alpha<\alpha_{0}$ there exists a unique infinite connected component of $\mathcal{N}_{\alpha}^{0}$, and its complement is composed of bounded connected sets.

With this proposition in mind, we may define clusters of regular Voronoi cells.

Definition 4.3 ( $\alpha$-clusters). Let $\alpha<\alpha_{0}$ be as in Proposition 4.2. We denote by $\mathcal{N}_{\alpha}$ the infinite connected component of $\mathcal{N}_{\alpha}^{0}$ defined therein. Moreover, we denote by $\mathcal{N}_{\alpha}^{*}$ the set of vertices of edges of $C_{i}$ with $i \in \mathcal{N}_{\alpha}$, by $\mathcal{V}_{\alpha}$ the set of the edges of such $C_{i}$, and by $\mathcal{E}_{\alpha}$ the set of edges of the Delaunay triangulation defined by set of pairs $(i, j)$ in $\mathcal{N}_{\alpha}^{2}$ such that $C_{i}$ and $C_{j}$ share a common edge.

Remark 4.4 (a channel property of $\mathcal{N}_{\alpha}$ ). With the notation of Definition 4.3 . note that the paths of cells $C_{i}$ in Lemma 4.1 can be taken with $i \in \mathcal{N}_{\alpha}$.

\subsection{Geometric properties of Voronoi tessellation of Pois- son set. Surface tension}

In this section we consider the geometric properties of the Poisson-Voronoi tessellation and introduce the surface tension in terms of an asymptotic distance between two points of the grid. In order to apply the subadditive theorem we should show that the grid distance between two arbitrary points has a finite expectation. The symbol $\mathbf{E}$ stands for the expectation in $\Omega$.

Proposition 4.5. For all $t>0$ we have

$$
\mathbf{E}\left(m_{0}((0,0),(t, 0))\right)<+\infty
$$

Furthermore, the limit

$$
\tau_{0}=\lim _{t \rightarrow+\infty} \frac{m_{0}((0,0),(t, 0))}{t}
$$

exists almost surely and is deterministic.

Proof. We say that a set $S \subset \mathbb{Z}^{2}$ is $l^{\infty}$-connected if for any two points $i$ and $j$ in $S$ there is a path $i=i_{0}, i_{1}, \ldots, i_{m}=j$ in $S$ such that $\left|i_{k}-i_{k-1}\right|_{\infty}=1$, $k=1, \ldots, m$.

Consider all $l^{\infty}$-connected sets in $\mathbb{Z}^{2}$ of size $n$ that contain the origin. According to [27, Proof of Theorem 4.20] for any $n \geq 0$ the number of such sets is not greater than $C_{2}^{n}$ for some constant $C_{2}>0$. 
Next we choose $L, K$ and $\alpha>0$ in such a way that $C_{2}^{2} \gamma<\frac{1}{4}$, where $\gamma$ is defined in (18).

We say that a site $j \in \mathbb{Z}^{2}$ is open if conditions $\left(\mathbf{c}_{1}\right)-\left(\mathbf{c}_{3}\right)$ in the proof of Lemma 4.1 are satisfied; otherwise $j$ is closed. The probability that a $l^{\infty}$-connected set in $\mathbb{Z}^{2}$ consists of closed points, has size $n$ and is a maximum $l^{\infty}$-connected component of closed points does not exceed $\gamma^{n}$. We denote such a set by $S(n)$.

Consider the sets

$$
\begin{gathered}
\mathcal{S}_{0}(n)=\bigcup_{j \in S(n)}\left(Q_{5 L}+10 L j\right), \\
\mathcal{S}_{1}(n)=\mathcal{S}_{0}(n) \bigcup\left\{x \in \mathbb{R}^{2}: \operatorname{dist}_{\infty}(x, 10 L S(n)) \leq 10 L\right\} .
\end{gathered}
$$

If $\mathcal{S}_{0}(n)$ contains $k$ points of $\mathcal{N}$, then the length of the shortest path from $(0,0)$ to $(1,0)$ does not exceed $(k+8 n K)^{2}$. The probability that $\mathcal{S}_{0}(n)$ contains exactly $k$ points of $\mathcal{N}$ is equal to

$$
\frac{\left(100 L^{2} n\right)^{k}}{k !} \exp \left(-100 L^{2} n\right) .
$$

Denote $L_{0}=100 L^{2}$.

The probability that $S(n)$ is a maximum connected component of closed sites and that $\mathcal{S}_{0}(n)$ contains exactly $k$ points of $\mathcal{N}$ is not greater than

$$
p_{k n}=\left(\gamma^{n}\right)^{\frac{1}{2}}\left(\frac{\left(L_{0} n\right)^{k}}{k !} \exp \left(-L_{0} n\right)\right)^{\frac{1}{2}} .
$$

Summing up over all connected sets in $\mathbb{Z}^{2}$ that contain the origin and over all $k$ from 0 to $+\infty$, we obtain that the expectation of the shortest path from $(0,0)$ to $(1,0)$ admits the following upper bound:

$$
\begin{aligned}
& \mathbf{E}\left(m_{0}((0,0),(1,0))\right) \leq \sum_{n=0}^{\infty} \sum_{k=0}^{\infty} C_{2}^{n} p_{k n}(k+8 n K)^{2} \\
\leq & \sum_{n, k=0}^{\infty} \exp \left(\left(\log \left(C_{2}\right)+\frac{1}{2} \log (\gamma)\right) n\right)\left(\frac{\left(L_{0} n\right)^{k}}{k !} \exp \left(-L_{0} n\right)\right)^{\frac{1}{2}}(k+8 n K)^{2} .
\end{aligned}
$$

Since $\frac{\left(L_{0} n\right)^{k}}{k !} \exp \left(-L_{0} n\right)<1$, using the Stirling formula and considering our choice of $\gamma$, one concludes that the series converges. This yields the relation in (19) for $t \leq 1$. For larger $t$ we use the subadditive property of $m_{0}((0,0),(t, 0))$. Namely, for any $s_{1}, t_{1}$ and $s_{2}, t_{2}$ we have

$$
m_{0}\left((0,0),\left(s_{2}, t_{2}\right)\right) \leq m_{0}\left((0,0),\left(s_{1}, t_{1}\right)\right)+m_{0}\left(\left(s_{1}, t_{1}\right),\left(s_{2}, t_{2}\right)\right) .
$$

This ensures the relation $\mathbf{E}\left(m_{0}((0,0),(t, 0))\right)<+\infty$ for any $t>0$.

The second statement now follows from the Kingman subadditive ergodic theorem, see [30] or 31] for details. 
Proposition 4.6 (isotropy and uniformity of the surface tension). We have

$$
\tau_{0}=\lim _{t \rightarrow+\infty} \frac{m_{0}(x, x+t v)}{t}
$$

for all $v \in S^{1}$, and the limit is uniform for $x=x(t)$ if $|x| \leq C t$ and $v \in S^{1}$.

Proof. Our first goal is to show that there exists a constant $C_{0}$ such that a.s. for any $\varkappa>0$ and $c_{1}>0$ and for all $t \geq t_{0}\left(\omega, c_{1}\right)$ we have

$$
m_{0}(x, y) \leq C_{0}|x-y|+\varkappa t+\sqrt{t}
$$

for all $x$ and $y$ from the cube $\left\{x \in \mathbb{R}^{2}:|x|_{\infty} \leq c_{1} t\right\}$. To this end we use again the definition of a cube $Q_{5 L}$ given in the proof of Lemma 4.1 and recall that a site $j \in \mathbb{Z}^{2}$ is open if conditions $\left(\mathbf{c}_{1}\right)-\left(\mathbf{c}_{3}\right)$ are fulfilled. We then choose the parameter $\gamma$ in (18) sufficiently small so that the open sites form a.s. an infinite open cluster that we call $\mathcal{C}$. Then a.s. for sufficiently large $t$ the diameter of any $l^{\infty}$-connected component of sites in in the complement to the infinite open cluster in $\left\{x \in \mathbb{R}^{2}:|x|_{\infty} \leq(10 L)^{-1} c_{1} t\right\}$ does not exceed $c_{2} \log t$ with $c_{2}>0$, see 27. Computing the probability to have in a cube of size $c_{2} \log t$ more than $\sqrt{t}$ points of $\mathcal{N}$, considering the fact that the number of such cubes centred at $j \in \mathbb{Z}^{2}$ and belonging to $\left\{x \in \mathbb{R}^{2}:|x|_{\infty} \leq(10 L)^{-1} c_{1} t\right\}$ grows polynomially in $t$ and using the Borel-Cantelli lemma we conclude that a.s. for sufficiently large $t$ we have

$$
m_{0}\left(x, \tilde{\pi}_{\alpha}(x)\right) \leq \sqrt{t}, \quad m_{0}\left(y, \tilde{\pi}_{\alpha}(y)\right) \leq \sqrt{t},
$$

where $\tilde{\pi}_{\alpha}(x)$ is the nearest to $x$ vertex of the union of the Voronoi cells that contain points of the scaled infinite open cluster $10 \mathrm{LC}$.

From the results in 25] it follows that a.s. for sufficiently large $t$, for any two points $j^{1}$ and $j^{2}$ of the open infinite cluster such that $j^{1}, j^{2} \in\left\{x \in \mathbb{R}^{2}\right.$ : $\left.|x|_{\infty} \leq(10 L)^{-1} c_{1} t\right\}$, and for any $\varkappa>0$ the cluster distance between $j^{1}$ and $j^{2}$ is not greater than $C_{2}\left|j^{1}-j^{2}\right|+\varkappa t$; here $C_{2}$ is a positive constant that does not depend on $\varkappa$. Combining this estimate with (22) we obtain (21).

Next, we are going to show that for any $x \in \mathbb{R}^{2}$ with $|x| \leq C$ and any $v \in S^{1}$ the limit relation

$$
\tau_{0}=\lim _{t \rightarrow+\infty} \frac{m_{0}(t x, t x+t v)}{t}
$$

holds a.s. In view of (21) it suffices to prove this relation for integer $t$ that tends to $\infty$. In the remaining part of the proof we call this parameter $n$ instead of $t$.

We fix a small positive $\theta>0$ and denote by $\mathcal{A}_{N}$ the event

$$
\mathcal{A}_{N}=\left\{\omega \in \Omega:\left|\frac{m_{0}(0, k v)}{k}-\tau_{0}\right| \leq \theta \text { for all } k \geq N\right\} .
$$

Since $\mathbf{P}\left(\mathcal{A}_{N}\right)$ tends to 1 as $N \rightarrow \infty$, for any $\delta>0$ there exists $N_{0}=N_{0}(\delta)$ such that

$$
\mathbf{P}\left(\mathcal{A}_{N_{0}}\right) \geq 1-\delta .
$$


By the Birkhoff ergodic theorem a.s. for any $\nu>0$ and $\varkappa>0$ there exists $k_{0}=k_{0}(\omega, \nu, \varkappa)$ such that

$$
\left|\frac{1}{k} \sum_{j=1}^{k} \mathbf{1}_{\mathcal{A}_{N_{0}}}\left(T_{j x} \omega\right)-\mathbf{P}\left(\mathcal{A}_{N_{0}}\right)\right| \leq \nu
$$

for all $k \geq \frac{1}{2} k_{0}$ and moreover inequality (21) holds for all such $k$. We assume that $\nu$ and $\delta$ are small enough so that $3(\nu+\delta) \leq \frac{1}{2}$.

For $k \geq k_{0}$ denote by $\ell$ the maximum of integers $j$ such that $j>k+1$ and for all $i \in(k, j)$ we have $T_{i x} \omega \notin \mathcal{A}_{N_{0}}$.

Let $M$ be the number of unities in the sequence $\left\{\boldsymbol{1}_{\mathcal{A}_{N_{0}}}\left(T_{i x} \omega\right)\right\}_{i=1}^{k}$. By the definition of $\ell$, the number of unities in $\left\{\mathbf{1}_{\mathcal{A}_{N_{0}}}\left(T_{i x} \omega\right)\right\}_{i=1}^{k+\ell}$ is equal to $M$ as well.

Since $k+\ell>k_{0}$, we have

$$
\nu>\left|\frac{M}{k+\ell}-\mathbf{P}\left(\mathcal{A}_{N_{0}}\right)\right|=\left|1-\mathbf{P}\left(\mathcal{A}_{N_{0}}\right)-\frac{\ell+(k-M)}{k+\ell}\right| .
$$

This yields

$$
\frac{\ell+(k-M)}{k+\ell}<\nu+1-\mathbf{P}\left(\mathcal{A}_{N_{0}}\right) \leq \nu+\delta .
$$

Since $k-M \geq 0$, recalling that $\nu+\delta \leq \frac{1}{6}$ we obtain $\ell \leq 2(\nu+\delta) k$.

For an arbitrary $k>\max \left(k_{0}, N_{0}\right)$ and $L=3(\nu+\delta) k$ there exists $n \in$ $[k, k+L]$ such that $T_{n x} \omega \in \mathcal{A}_{N_{0}}$. Then we have

$$
\left|\frac{1}{k} m_{0}^{\omega}(n x, n x+k v)-\tau_{0}\right|=\left|\frac{1}{k} m_{0}^{T_{n x} \omega}(0, k v)-\tau_{0}\right| \leq \theta .
$$

Since $n-k \leq 3(\nu+\delta) k$ and $k>k_{0}$, then by (21)

$$
\left|m_{0}(n x, n x+k v)-m_{0}(k x, k x+k v)\right| \leq\left[3 C_{0} C(\nu+\delta)+\varkappa\right] k+\sqrt{k} .
$$

Dividing by $k$ and considering (24) we obtain

$$
\left|\frac{1}{k} m_{0}^{\omega}(k x, k x+k v)-\tau_{0}\right| \leq \theta+\left[3 C_{0} C(\nu+\delta)+\varkappa\right]+\frac{1}{\sqrt{k}} .
$$

It remains to take into account the fact that $\theta, \nu, \delta$ and $\varkappa$ are arbitrary positive number, and (23) follows.

In view of estimate (21) the pointwise convergence in (23) implies the uniform convergence in (20) for $|x| \leq C t$. This completes the proof.

Proposition 4.7 (coerciveness of the surface tension). We have $\tau_{0}>0$.

Proof. Given $t>0$ take a minimal path $\left\{e_{k}\right\}$ for $m_{0}((0,0),(0, t))$. We can apply Lemma 3.1 with $\varepsilon=1 / t, R=1, \gamma=1 / 2$, and $P \in \Pi$ with $e_{k} \subset P$ for all $k$. We then have

$$
t(1+o(1)) \leq \# \mathbf{A}(P) \leq C \#\left\{C_{i}: C_{i} \subset P\right\} \leq C \#\left\{e_{k}\right\},
$$

which shows the claim, since the constant in this estimate are independent of $t$. 
Proposition 4.8. There exists a constant $C_{0}$ such that if $t$ is large enough then if $\left\{e_{k}\right\}$ is a test path for $m_{0}(x, x+t v)$ with $x$ as in Proposition 4.6 with $\#\left(\left\{e_{k}\right\}\right) \leq t M$, then each point of $\left\{e_{k}\right\}$ is at most at distance $C_{0} M t$ from $x$.

Proof. It suffices to apply Lemma 3.1 to the set of all Voronoi cells with non empty intersection with $\bigcup_{k} e_{k}$ and $\varepsilon=1 / t$. We then cover $\bigcup_{k} e_{k}$ with the union of at most $2 C_{0} M t$ cubes, from which the claim follows.

\subsection{Computation of the $\Gamma$-limit}

Lower bound. We use an argument typical of the blow-up technique [24, 16].

Let $u^{\varepsilon} \rightarrow A$. Since $A$ is of finite perimeter, with fixed $\sigma>0$ and $\delta>0$ we consider a disjoint finite family of rectangles

$$
R_{i}=\left\{x:\left|\left\langle x-x_{i}, \nu_{i}\right\rangle\right| \leq \delta \rho_{i},\left|\left\langle x-x_{i}, \nu_{i}^{\perp}\right\rangle\right| \leq \frac{1}{2} \rho_{i}\right\}
$$

such that

$$
\mathcal{H}^{1}\left(\partial A \backslash \bigcup_{i} R_{i}\right) \leq \sigma \quad \text { and } \quad\left|\sum_{i} \rho_{i}-\mathcal{H}^{1}(\partial A)\right| \leq \sigma .
$$

Since $A_{\varepsilon} \rightarrow A$ we may assume that

$$
\mathcal{L}^{2}\left(A_{\varepsilon} \cap R_{i}^{+}\right)=o(1), \quad \mathcal{L}^{2}\left(\left(A \backslash A_{\varepsilon}\right) \cap R_{i}^{+}\right)=o(1)
$$

as $\varepsilon \rightarrow 0$, where

$$
R_{i}^{ \pm}=R_{i} \pm 2 \delta \rho_{i} \nu_{i}
$$

We now fix an index $i$. We use the channel property in Lemma 4.1 to find a path $\left\{\varepsilon C_{j}^{+}\right\}$joining the two sides of $R_{i}^{+}$parallel to $\nu_{i}$, with $j$ endpoints of segments of a path in $\mathcal{E}_{\alpha}$, and such that

$$
\mathcal{L}^{2}\left(A_{\varepsilon} \cap R_{i}^{+} \cap \varepsilon \bigcup_{j} C_{j}^{+}\right) \leq \frac{\varepsilon}{C_{\delta} \rho_{i}} \mathcal{L}^{2}\left(A_{\varepsilon} \cap R_{i}^{+}\right),
$$

which follows from the existence of a number of disjoints paths proportional to $\rho_{i}$.

Note that, since $\left|\varepsilon C_{j}^{+}\right| \geq \pi \varepsilon^{2} \alpha^{2}$, we have

$$
\#\left\{j: \varepsilon C_{j}^{+} \subset A_{\varepsilon}\right\} \leq \frac{1}{\pi \varepsilon^{2} \alpha^{2}} \mathcal{L}^{2}\left(A_{\varepsilon} \cap R_{i}^{+} \cap \varepsilon \bigcup_{j} C_{j}^{+}\right) \leq \frac{1}{\varepsilon \pi C_{\delta} \rho_{i} \alpha^{2}} \mathcal{L}^{2}\left(A_{\varepsilon} \cap R_{i}^{+}\right) .
$$

Similarly, we define $\left\{C_{j}^{-}\right\}$joining the two sides of $R_{i}^{-}$parallel to $\nu_{i}$, and such that

$$
\mathcal{L}^{2}\left(\left(A \backslash A_{\varepsilon}\right) \cap R_{i}^{-} \cap \varepsilon \bigcup_{j} C_{j}^{-}\right) \leq \frac{\varepsilon}{C_{\delta} \rho_{i}} \mathcal{L}^{2}\left(\left(A \backslash A_{\varepsilon}\right) \cap R_{i}^{-}\right),
$$


so that

$$
\#\left\{j: \varepsilon C_{j}^{-} \subset\left(A \backslash A_{\varepsilon}\right)\right\} \leq \frac{1}{\varepsilon \pi C_{\delta} \rho_{i} \alpha^{2}} \mathcal{L}^{2}\left(\left(\left(A \backslash A_{\varepsilon}\right) \cap R_{i}^{-}\right) .\right.
$$

We define $U_{\varepsilon}^{+}$as the connected component of $R_{i}^{+} \backslash \varepsilon \bigcup_{j} C_{j}^{+}$containing the upper side $S_{i}^{+}=\left\{x \in R_{i}^{+}:\left\langle x-x_{i}, \nu_{i}\right\rangle=3 \delta \rho_{i}\right\}$ and $U_{\varepsilon}^{-}$as the connected component of $R_{i}^{-} \backslash \varepsilon \bigcup_{j} C_{j}^{-}$containing the lower side $S_{i}^{-}=\left\{x \in R_{i}^{-}:\langle x-\right.$ $\left.\left.x_{i}, \nu_{i}\right\rangle=-3 \delta \rho_{i}\right\}$, and define

$$
\widetilde{A}_{\varepsilon}=\left(A_{\varepsilon} \backslash U_{\varepsilon}^{+}\right) \cup U_{\varepsilon}^{-} .
$$

We now consider the connected component of the set $\left(R_{i} \cup R_{i}^{+} \cup R_{i}^{-}\right) \backslash \widetilde{A}_{\varepsilon}$ containing the upper side $S_{i}^{+}$. Note that this connected component does not contain $S_{i}^{-}$, so that it contains a path of edges $\left\{e_{k}^{\varepsilon}\right\}$ in $\mathcal{V}$ connecting the two sides of $R_{i} \cup R_{i}^{+} \cup R_{i}^{-}$parallel to $\nu_{i}$. We denote by $x_{\varepsilon}^{ \pm}$the extreme points of this path.

Using Proposition 4.6, we can now estimate

$$
\begin{aligned}
\#\left\{\text { edges of } \partial V_{\varepsilon}\left(u^{\varepsilon}\right) \text { inside } R_{i}\right\} & \geq \#\left\{\text { edges of } \partial A_{\varepsilon} \text { inside } R_{i}\right\} \\
& \geq \#\left\{e_{k}^{\varepsilon}\right\}-\frac{1}{\varepsilon \pi C_{\delta} \rho_{i} \alpha^{2}} o(1) \\
& \geq m_{0}\left(x_{\varepsilon}^{-}, x_{\varepsilon}^{+}\right)-\frac{1}{\varepsilon \pi C_{\delta} \rho_{i} \alpha^{2}} o(1) \\
& \geq\left(\tau_{0}+o(1)\right) \frac{\rho_{i}}{\varepsilon}-\frac{1}{\varepsilon \pi C_{\delta} \rho_{i} \alpha^{2}} o(1) .
\end{aligned}
$$

Summing up in $i$ we then get

$$
\liminf _{\varepsilon \rightarrow 0} E_{\varepsilon}\left(u^{\varepsilon}\right) \geq \sum_{i} \rho_{i} \tau_{0} \geq \tau_{0}\left(\mathcal{H}^{1}(\partial A)-\sigma\right)
$$

and prove the claim by the arbitrariness of $\sigma$.

Upper bound. By an approximation argument [11, 10] it is sufficient to prove the upper bound for polyhedral sets. Moreover, we can just deal with a single connected bounded polyhedron $A$ with a connected boundary since all other cases can be reduced to that by considering union or complements of such sets.

We write the boundary of $A$ as the union of segments $\left[x_{j-1}, x_{j}\right]$ with endpoints $x_{0}, \ldots, x_{N} \in \mathbb{R}^{2}$ with $x_{N}=x_{0}$. With fixed $m \in \mathbb{N}$ and $\delta>0$, for all $j \in\{1, \ldots, N\}$ and $l \in\{1, \ldots, m\}$ we consider a non-intersecting path $\left\{e_{k}^{j, l}\right\}$ in $\mathcal{V}$ between $\pi_{0}\left(x_{j, m-1}^{\varepsilon}\right)$ and $\pi_{0}\left(x_{j, m}^{\varepsilon}\right)$, where

$$
x_{j, m}^{\varepsilon}=\frac{1}{\varepsilon}\left(x_{j-1}+\frac{l}{m}\left(x_{j}-x_{j-1}\right)\right),
$$

such that

$$
\#\left\{e_{k}^{j, l}\right\} \leq \frac{1}{m \varepsilon}\left|x_{j}-x_{j-1}\right|\left(\tau_{0}+\delta\right) .
$$


Denoting the union of the rescaled paths

$$
B_{\varepsilon}^{\delta, m}=\varepsilon \bigcup_{j, l, k} e_{k}^{j, l}
$$

let $A_{\varepsilon}^{\delta, m}$ be the complement of the infinite connected component of $\mathbb{R}^{2} \backslash B_{\varepsilon}^{\delta, m}$ (note that the paths $\left\{e_{k}^{j, l}\right\}$ may intersect, so that there may be more than one bounded connected component of the complement of their union). If $u^{\varepsilon}$ is defined as

$$
u_{i}^{\varepsilon}= \begin{cases}1 & \text { if } i \in A_{\varepsilon}^{\delta, m} \\ -1 & \text { if } i \notin A_{\varepsilon}^{\delta, m}\end{cases}
$$

then we have

$$
\begin{aligned}
E_{\varepsilon}\left(u^{\varepsilon}\right) & \leq \varepsilon \sum_{j, l, k} \#\left\{e_{k}^{j, l}\right\} \leq \sum_{j, l} \frac{1}{m}\left|x_{j}-x_{j-1}\right|\left(\tau_{0}+\delta\right) \\
& =\mathcal{H}^{1}(\partial A)\left(\tau_{0}+\delta\right),
\end{aligned}
$$

since the boundary of $A_{\varepsilon}^{\delta, m}$ is contained in $B_{\varepsilon}^{\delta, m}$.

By Lemma 2.2. thanks to (27) these sets converge as $\varepsilon \rightarrow 0$ to a set of finite perimeter $A^{\delta, m}$, and

$$
\Gamma-\limsup _{\varepsilon \rightarrow 0} E_{\varepsilon}\left(A^{\delta, m}\right) \leq \mathcal{H}^{1}(\partial A)\left(\tau_{0}+\delta\right) .
$$

Thanks to Proposition 4.8 each point of $\varepsilon\left\{e_{k}^{j, l}\right\}$ is at most at a distance $C / m$ from the segment $\left[\varepsilon \pi_{0}\left(x_{j, m-1}^{\varepsilon}\right), \varepsilon \pi_{0}\left(x_{j, m}^{\varepsilon}\right)\right]$, and hence, since

$$
\lim _{\varepsilon \rightarrow 0} \varepsilon \pi_{0}\left(x_{j, m}^{\varepsilon}\right)=x_{j-1}+\frac{l}{m}\left(x_{j}-x_{j-1}\right),
$$

the boundary of $A^{\delta, m}$ is contained in a $C / m$-neighbourhood of $\partial A$. This implies that $A^{\delta, m}$ converge to $A$ as $m \rightarrow+\infty$ independently of $\delta$. By the lower semicontinuity of the $\Gamma$-limsup [1] we then deduce that

$$
\Gamma-\limsup _{\varepsilon \rightarrow 0} E_{\varepsilon}(A) \leq \lim _{m \rightarrow+\infty} \Gamma-\limsup _{\varepsilon \rightarrow 0} E_{\varepsilon}\left(A^{\delta, m}\right) \leq \mathcal{H}^{1}(\partial A)\left(\tau_{0}+\delta\right),
$$

and the claim is proved.

\section{Approximate surface tensions}

In this final section we consider the restriction of the energies $E_{\varepsilon}$ to (spin functions with related) sets whose boundary is composed of edges of $\alpha$-regular Voronoi cells. We denote by $E_{\varepsilon}^{\alpha}$ such energies. Note that in this case $E_{\varepsilon}^{\alpha}\left(u^{\varepsilon}\right)$ immediately gives the equiboundedness of the perimeter of the sets $V_{\varepsilon}\left(u^{\varepsilon}\right)$ and hence their precompactness. We briefly describe the limit of $E_{\varepsilon}^{\alpha}$ at fixed $\alpha$. 
With given $\alpha<\alpha_{0}$ as in Proposition 4.2 we define for all $x \in \mathbb{R}^{2}$

$$
\pi_{\alpha}(x)=\text { closest point of } \mathcal{N}_{\alpha}^{*} \text { to } x .
$$

For almost all $x$ this point is uniquely defined. For the remaining points we choose one of the closest points of $\mathcal{N}_{\alpha}^{*}$ to $x$. For all $x, y \in \mathbb{R}^{2}$ we set

$$
m_{\alpha}(x, y)=\min \left\{\#\left\{e_{k}\right\}:\left\{e_{k}\right\} \text { is a path in } \mathcal{V}_{\alpha} \text { connecting } \pi_{\alpha}(x) \text { and } \pi_{\alpha}(y)\right\} .
$$

Proposition 5.1. For all $\alpha<\alpha_{0}$ a.s. the limit

$$
\tau_{\alpha}=\lim _{t \rightarrow+\infty} \frac{m_{\alpha}(x, x+t v)}{t}
$$

exists for all $v \in S^{1}$, and the limit is uniform for $x=x(t)$ if $|x| \leq C t$ and $v \in S^{1}$. Furthermore $\tau_{\alpha} \in(0,+\infty)$.

Proof. The proof follows that for $\tau_{0}$, and is actually simpler as bounds for $m_{\alpha}(x, x+t v)$ are easier

Theorem 5.2 (homogenization on the $\alpha$-cluster). For $\alpha<\alpha_{0}$ almost surely there exists the $\Gamma$-limit of $E_{\varepsilon}^{\alpha}$ and it equals $\tau_{\alpha} \mathcal{H}^{1}(\partial A)$.

Proof. The proof is the same as for the homogenization theorem in the previous section, taking care of using the same $\alpha$ as the one labeling the energies in the proof of the lower bound. Conversely, for the proof of the upper inequality, it is not necessary to use Proposition 4.8 .

Proposition 5.3. We have $\inf _{\alpha<\alpha_{0}} \tau_{\alpha}=\lim _{\alpha \rightarrow 0} \tau_{\alpha}$.

Proof. Choose $\alpha_{0}>0$ in such a way that for some $L$ and $K$ we have

$$
\mathbf{P}\left(\mathcal{E}\left(L, K, \alpha_{0}, j\right)\right)>p_{\text {cr. }} .
$$

It suffices to show that $\tau_{\alpha_{1}} \leq \tau_{\alpha_{2}}$, if $0<\alpha_{1}<\alpha_{2} \leq \alpha_{0}$. Since $\mathcal{N}_{\alpha_{2}}^{*} \subset \mathcal{N}_{\alpha_{1}}^{*}$, then

$$
\min \left\{\#\left\{e_{k}\right\}:\left\{e_{k}\right\} \text { is a path in } \mathcal{V}_{\alpha_{1}} \text { connecting } \pi_{\alpha_{2}}\left(x_{0}\right) \text { and } \pi_{\alpha_{2}}\left(x_{t}\right)\right\}
$$

$$
\leq m_{\alpha_{2}}\left(x_{0}, x_{t}\right)
$$

where $x_{0}=0$ and $x_{t}=(t, 0)$. We should estimate

$$
\min \left\{\#\left\{e_{k}\right\}:\left\{e_{k}\right\} \text { is a path in } \mathcal{V}_{\alpha_{1}} \text { connecting } \pi_{\alpha_{1}}\left(x_{t}\right) \text { and } \pi_{\alpha_{2}}\left(x_{t}\right)\right\} \text {. }
$$

To this end we consider the cubes $Q_{5 L}+10 L j, j \in \mathbb{Z}^{2}$, that were introduced in the proof of Lemma 4.1 and take those of them that satisfy conditions $\left(\mathbf{c}_{1}\right)-\left(\mathbf{c}_{3}\right)$ for $\alpha=\alpha_{0}$. Under our choice of $\alpha_{0}$ a.s. these exists a unique infinite cluster of such cubes. The complement to the infinite cluster consists of connected bounded sets. Moreover, according to [27, for sufficiently large $t$ the maximal size of the connected components in the complement to the infinite cluster that 
have a non-trivial intersection with $\left.[-2 t, 2 t]^{2}\right]$ does not exceed $c \log (t)$. This implies that the size of the maximal connected component of $\left.[-2 t, 2 t]^{2}\right] \backslash \mathcal{V}_{\alpha_{0}}$ does not exceed $c \log (t)$. Since $\mathcal{N}_{\alpha_{0}}^{*} \subset \mathcal{N}_{\alpha_{2}}^{*} \subset \mathcal{N}_{\alpha_{1}}^{*}$, then $\pi_{\alpha_{1}}\left(x_{t}\right)$ and $\pi_{\alpha_{2}}\left(x_{t}\right)$ belong to the closure of the same connected component of $\left.[-2 t, 2 t]^{2}\right] \backslash \mathcal{V}_{\alpha_{0}}$. Therefore,

$\lim _{t \rightarrow \infty} \frac{1}{t} \min \left\{\#\left\{e_{k}\right\}:\left\{e_{k}\right\}\right.$ is a path in $\mathcal{V}_{\alpha_{1}}$ connecting $\pi_{\alpha_{1}}\left(x_{t}\right)$ and $\left.\pi_{\alpha_{2}}\left(x_{t}\right)\right\}=0$.

Similarly,

$\lim _{t \rightarrow \infty} \frac{1}{t} \min \left\{\#\left\{e_{k}\right\}:\left\{e_{k}\right\}\right.$ is a path in $\mathcal{V}_{\alpha_{1}}$ connecting $\pi_{\alpha_{1}}\left(x_{0}\right)$ and $\left.\pi_{\alpha_{2}}\left(x_{0}\right)\right\}=0$.

Combining these two relations with (29) we obtain the required inequality $\tau_{\alpha_{1}} \leq$ $\tau_{\alpha_{2}}$.

\section{Acknowledgments.}

The authors acknowledge the MIUR Excellence Department Project awarded to the Department of Mathematics, University of Rome Tor Vergata, CUP E83C18000100006.

\section{References}

[1] R. Alicandro, A. Braides, and M. Cicalese, Phase and anti-phase boundaries in binary discrete systems: a variational viewpoint. Netw. Heterog. Media 1 (2006), 85-107

[2] R. Alicandro, M. Cicalese, and A. Gloria. Integral representation results for energies defined on stochastic lattices and application to nonlinear elasticity. Arch. Ration. Mech. Anal. 200 (2011), 881-943.

[3] R. Alicandro, M. Cicalese, and M. Ruf. Domain formation in magnetic polymer composites: an approach via stochastic homogenization. Arch. Ration. Mech. Anal. 218 (2015), 945-984.

[4] L. Ambrosio. Existence theory for a new class of variational problems. Arch. Ration. Mech. Anal. 111 (1990), 291-322

[5] L. Ambrosio and A. Braides, Functionals defined on partitions of sets of finite perimeter, II: semicontinuity, relaxation and homogenization, J. Math. Pures. Appl. 69 (1990), 307-333.

[6] A. Bach, A. Braides, and M. Cicalese. Discrete-to-continuum limits of multi-body systems with bulk and surface long-range interactions. Preprint 2019. 
[7] A. Bach, A. Braides and C. I. Zeppieri. Quantitative analysis of finitedifference approximations of free-discontinuity problems. Preprint 2018.

[8] X. Blanc, C. Le Bris, and P.L. Lions. The energy of some microscopic stochastic lattices. Arch. Ration. Mech. Anal. 184 (2007), 303-339.

[9] T. Bodineau, D. Ioffe, Y. Velenik, Rigorous probabilistic analysis of equilibrium crystal shapes, J. Math. Phys. 41 (2000) 1033-1098.

[10] A. Braides, Approximation of Free-Discontinuity Problems, Lecture Notes in Mathematics 1694, Springer Verlag, Berlin, 1998.

[11] A. Braides, Г-convergence for Beginners, Oxford University Press, Oxford, 2002.

[12] A. Braides, A handbook of $\Gamma$-convergence. In Handbook of Differential Equations. Stationary Partial Differential Equations, Volume 3 (M. Chipot and P. Quittner, eds.), Elsevier, 2006.

[13] A. Braides, M. Cicalese, and M. Ruf. Continuum limit and stochastic homogenization of discrete ferromagnetic thin films. Anal. PDE 11 (2018), 499-553.

[14] A. Braides, A. Defranceschi and E. Vitali. Homogenization of free discontinuity problems. Arch. Ration. Mech. Anal. 135 (1996), 297-356.

[15] A. Braides and L. Kreutz. An integral-representation result for continuum limits of discrete energies with multi-body interactions. SIAM J. Math. Anal. 50 (2018), 1485-1520

[16] A. Braides, M. Maslennikov, and L. Sigalotti. Homogenization by blow-up. Applicable Anal. 87 (2008), 1341-1356.

[17] A. Braides and A. Piatnitski, Overall properties of a discrete membrane with randomly distributed defects. Arch. Ration. Mech. Anal. 189 (2008), 301-323.

[18] A. Braides and A. Piatnitski. Variational problems with percolation: dilute spin systems at zero temperature J. Stat. Phys. 149 (2012), 846-864

[19] A. Braides and A. Piatnitski. Homogenization of surface and length energies for spin systems. J. Funct. Anal. 264 (2013), 1296-1328.

[20] L.A. Caffarelli and R. de la Llave. Planelike minimizers in periodic media. Comm. Pure Appl. Math. 54 (2001), 1403-1441.

[21] F. Cagnetti, G. Dal Maso, L. Scardia, and C. I. Zeppieri. Г-convergence of free-discontinuity problems. Ann. Inst. H. Poincaré Anal. Non Linéaire 36 (2019), 1035-1079. 
[22] P. Calka. Precise formulae for the distributions of the principal geometric characteristics of the typical cells of a two-dimensional Poisson-Voronoi tessellation and a Poisson line process. Adv. Appl. Prob. 35 (2003), 551562.

[23] D. J. Daley and D. Vere-Jones, An introduction to the theory of point processes, Springer-Verlag, New York, 1988.

[24] I. Fonseca and S. Müller. Quasi-convex Integrands and lower semicontinuity in $L^{1}$. SIAM J. Math. Anal. 23 (1992), 1081-1098.

[25] O. Garet and R. Marchand, Asymptotic shape for the chemical distance and first passage percolation on the infinite Bernoulli cluster. ESAIM Probab. Statist. 8 (2004), 169-199.

[26] O. Garet and R. Marchand, Large deviations for the chemical distance in supercritical Bernoulli percolation. Annals of Probability 35 (2007), 833866.

[27] G. Grimmet. Percolation. Springer, Berlin, 1999.

[28] V.V. Jikov; S.M. Kozlov; O.A. Oleinik, Homogenization of differential operators and integral functionals., Springer-Verlag, Berlin, 1994.

[29] H. Kesten, Percolation Theory for Mathematicians. Progress in Probability and Statistics, 2. Birkhäuser, Boston, 1982

[30] JFC. Kingman. Subadditive ergodic theory. The Annals of Probability, 1 (1973), 883-899.

[31] U. Krengel. Ergodic Theorems. W. de Gruyter, Berlin, 2011.

[32] U. Krengel and R. Pyke, Uniform pointwise ergodic theorems for classes of averaging sets and multiparameter subadditive processes. Stoch. Proc. Appl. 26 (1987) 298-296.

[33] F. Maggi. Sets of Finite Perimeter and Geometric Variational Problems: an Introduction to Geometric Measure Theory. Cambridge University Press, Cambridge, 2012.

[34] L.P.R. Pimentel. On some fundamental aspects of polyominoes on random Voronoi tilings. Brazilian Journal of Probability and Statistics 27 (2013), $54-69$ 